

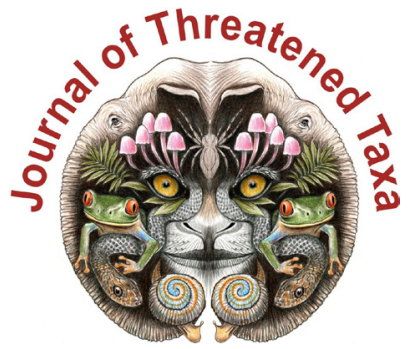

ISSN 0974-7907 (Online); ISSN $0974-7893$ (Print)

Publisher

Host

Wildlife Information Liaison Development Society

www.wild.zooreach.org

Zoo Outreach Organization www.zooreach.org

No. 12, Thiruvannamalai Nagar, Saravanampatti - Kalapatti Road, Saravanampatti, Coimbatore, Tamil Nadu 641035, India

Ph: +91 9385339863 | www.threatenedtaxa.org

Email: sanjay@threatenedtaxa.org

EDITORS

\section{Founder \& Chief Editor}

Dr. Sanjay Molur

Wildlife Information Liaison Development (WILD) Society \& Zoo Outreach Organization (ZOO),

12 Thiruvannamalai Nagar, Saravanampatti, Coimbatore, Tamil Nadu 641035, India

\section{Deputy Chief Editor}

Dr. Neelesh Dahanukar

Noida, Uttar Pradesh, India

\section{Managing Editor}

Mr. B. Ravichandran, WILD/ZOO, Coimbatore, India

\section{Associate Editors}

Dr. Mandar Paingankar, Government Science College Gadchiroli, Maharashtra 442605, India

Dr. Ulrike Streicher, Wildlife Veterinarian, Eugene, Oregon, USA

Ms. Priyanka Iyer, ZOO/WILD, Coimbatore, Tamil Nadu 641035, India

Dr. B.A. Daniel, ZOO/WILD, Coimbatore, Tamil Nadu 641035, India

\section{Editorial Board}

Dr. Russel Mittermeier

Executive Vice Chair, Conservation International, Arlington, Virginia 22202, USA

\section{Prof. Mewa Singh Ph.D., FASc, FNA, FNASc, FNAPsy}

Ramanna Fellow and Life-Long Distinguished Professor, Biopsychology Laboratory, and Institute of Excellence, University of Mysore, Mysuru, Karnataka 570006, India; Honorary Professor, Jawaharlal Nehru Centre for Advanced Scientific Research, Bangalore; and Adjunct Professor, National Institute of Advanced Studies, Bangalore

\section{Stephen D. Nash}

Scientific Illustrator, Conservation International, Dept. of Anatomical Sciences, Health Sciences Center, T-8, Room 045, Stony Brook University, Stony Brook, NY 11794-8081, USA

\section{Dr. Fred Pluthero}

Toronto, Canada

\section{Dr. Priya Davidar}

Sigur Nature Trust, Chadapatti, Mavinhalla PO, Nilgiris, Tamil Nadu 643223, India

\section{Dr. Martin Fisher}

Senior Associate Professor, Battcock Centre for Experimental Astrophysics, Cavendish

Laboratory, JJ Thomson Avenue, Cambridge CB3 OHE, UK

\section{Dr. John Fellowes}

Honorary Assistant Professor, The Kadoorie Institute, 8/F, T.T. Tsui Building, The University of Hong Kong, Pokfulam Road, Hong Kong

\section{Prof. Dr. Mirco Solé}

Universidade Estadual de Santa Cruz, Departamento de Ciências Biológicas, Vice-coordenado do Programa de Pós-Graduação em Zoologia, Rodovia Ilhéus/Itabuna, Km 16 (45662-000)

Salobrinho, Ilhéus - Bahia - Brasil

\section{Dr. Rajeev Raghavan}

Professor of Taxonomy, Kerala University of Fisheries \& Ocean Studies, Kochi, Kerala, India

\section{English Editors}

Mrs. Mira Bhojwani, Pune, India

Dr. Fred Pluthero, Toronto, Canad

Mr. P. Ilangovan, Chennai, India

Web Development

Mrs. Latha G. Ravikumar, ZOO/WILD, Coimbatore, India

\section{Typesetting}

Mr. Arul Jagadish, ZOO, Coimbatore, India

Mrs. Radhika, ZOO, Coimbatore, India

Mrs. Geetha, ZOO, Coimbatore India
Fundraising/Communications

Mrs. Payal B. Molur, Coimbatore, India

Subject Editors 2018-2020

Fungi

Dr. B. Shivaraju, Bengaluru, Karnataka, India

Dr. R.K. Verma, Tropical Forest Research Institute, Jabalpur, India

Dr. Vatsavaya S. Raju, Kakatiay University, Warangal, Andhra Pradesh, India

Dr. M. Krishnappa, Jnana Sahyadri, Kuvempu University, Shimoga, Karnataka, India

Dr. K.R. Sridhar, Mangalore University, Mangalagangotri, Mangalore, Karnataka, India

Dr. Gunjan Biswas, Vidyasagar University, Midnapore, West Bengal, India

\section{Plants}

Dr. G.P. Sinha, Botanical Survey of India, Allahabad, India

Dr. N.P. Balakrishnan, Ret. Joint Director, BSI, Coimbatore, India

Dr. Shonil Bhagwat, Open University and University of Oxford, UK

Prof. D.J. Bhat, Retd. Professor, Goa University, Goa, India

Dr. Ferdinando Boero, Università del Salento, Lecce, Italy

Dr. Dale R. Calder, Royal Ontaro Museum, Toronto, Ontario, Canada

Dr. Cleofas Cervancia, Univ. of Philippines Los Baños College Laguna, Philippines

Dr. F.B. Vincent Florens, University of Mauritius, Mauritius

Dr. Merlin Franco, Curtin University, Malaysia

Dr. V. Irudayaraj, St. Xavier's College, Palayamkottai, Tamil Nadu, India

Dr. B.S. Kholia, Botanical Survey of India, Gangtok, Sikkim, India

Dr. Pankaj Kumar, Kadoorie Farm and Botanic Garden Corporation, Hong Kong S.A.R., China

Dr. V. Sampath Kumar, Botanical Survey of India, Howrah, West Bengal, India

Dr. A.J. Solomon Raju, Andhra University, Visakhapatnam, India

Dr. Vijayasankar Raman, University of Mississippi, USA

Dr. B. Ravi Prasad Rao, Sri Krishnadevaraya University, Anantpur, India

Dr. K. Ravikumar, FRLHT, Bengaluru, Karnataka, India

Dr. Aparna Watve, Pune, Maharashtra, India

Dr. Qiang Liu, Xishuangbanna Tropical Botanical Garden, Yunnan, China

Dr. Noor Azhar Mohamed Shazili, Universiti Malaysia Terengganu, Kuala Terengganu, Malaysia

Dr. M.K. Vasudeva Rao, Shiv Ranjani Housing Society, Pune, Maharashtra, India

Prof. A.J. Solomon Raju, Andhra University, Visakhapatnam, India

Dr. Mandar Datar, Agharkar Research Institute, Pune, Maharashtra, India

Dr. M.K. Janarthanam, Goa University, Goa, India

Dr. K. Karthigeyan, Botanical Survey of India, India

Dr. Errol Vela, University of Montpellier, Montpellier, France

Dr. P. Lakshminarasimhan, Botanical Survey of India, Howrah, India

Dr. Larry R. Noblick, Montgomery Botanical Center, Miami, USA

Dr. K. Haridasan, Pallavur, Palakkad District, Kerala, India

Dr. Analinda Manila-Fajard, University of the Philippines Los Banos, Laguna, Philippines

Dr. P.A. Sinu, Central University of Kerala, Kasaragod, Kerala, India

Dr. Afroz Alam, Banasthali Vidyapith (accredited A grade by NAAC), Rajasthan, India

Dr. K.P. Rajesh, Zamorin's Guruvayurappan College, GA College PO, Kozhikode, Kerala, India

Dr. David E. Boufford, Harvard University Herbaria, Cambridge, MA 02138-2020, USA

Dr. Ritesh Kumar Choudhary, Agharkar Research Institute, Pune, Maharashtra, India

Dr. Navendu Page, Wildlife Institute of India, Chandrabani, Dehradun, Uttarakhand, India

\section{Invertebrates}

Dr. R.K. Avasthi, Rohtak University, Haryana, India

Dr. D.B. Bastawade, Maharashtra, India

Dr. Partha Pratim Bhattacharjee, Tripura University, Suryamaninagar, India

Dr. Kailash Chandra, Zoological Survey of India, Jabalpur, Madhya Pradesh, India

Dr. Ansie Dippenaar-Schoeman, University of Pretoria, Queenswood, South Africa

Dr. Rory Dow, National Museum of natural History Naturalis, The Netherlands

Dr. Brian Fisher, California Academy of Sciences, USA

Dr. Richard Gallon, llandudno, North Wales, LL30 1UP

Dr. Hemant V. Ghate, Modern College, Pune, India

Dr. M. Monwar Hossain, Jahangirnagar University, Dhaka, Bangladesh

Mr. Jatishwor Singh Irungbam, Biology Centre CAS, Branišovská, Czech Republic.

Dr. Ian J. Kitching, Natural History Museum, Cromwell Road, UK

Dr. George Mathew, Kerala Forest Research Institute, Peechi, India

For Focus, Scope, Aims, and Policies, visit https://threatenedtaxa.org/index.php/JoTT/aims_scope
For Article Submission Guidelines, visit https://threatenedtaxa.org/index.php/JoTT/about/submissions
For Policies against Scientific Misconduct, visit https://threatenedtaxa.org/index.php/JoTT/policies_various

continued on the back inside cover 


\title{
Diversity of aquatic insects and biomonitoring of water quality in the upper Ganga River, a Ramsar site: a preliminary assessment
}

\author{
Kritish De ${ }^{1} \mathbb{D}$, Arkojyoti Sarkar ${ }^{2}$ (D) Kritika Singh ${ }^{3}$ D , Virendra Prasad Uniyal ${ }^{4}$ (D), \\ Jeyaraj Antony Johnson ${ }^{5}$ (D) \& Syed Ainul Hussain ${ }^{6} \mathbb{D}$
}

${ }^{1-6}$ Wildlife Institute of India, Chandrabani, Dehradun, Utarakhand 248001, India.

${ }^{1}$ Present address: Department of Life Sciences, Sri Sathya Sai University for Human Excellence, Navanihal, Okali Post, Kamalapur, Karnataka 585313, India.

${ }^{1}$ kritish.de@gmail.com (corresponding author), ${ }^{2}$ arko.joti777@gmail.com, ${ }^{3}$ kritika17singh@gmail.com, ${ }^{4}$ uniyalvp@wii.gov.in, ${ }^{5}$ jaj@wii.gov.in, ${ }^{6}$ hussainsyedainul@wii.gov.in

\begin{abstract}
Monitoring of freshwater habitats through aquatic insects is widely used. A study was carried out in March, 2019 at 14 sites in the Upper Ganga River between Brijghat and Narora, a riverine Ramsar site in India, to document the diversity of three major aquatic predatory insect groups-Odonata, Coleoptera, and Hemiptera-and determine their biomonitoring potential. The study recorded three species of Coleoptera, four Hemiptera, 14 dragonflies, and eight damselflies. The Shannon diversity index ( $\mathrm{H}^{\prime}$ ) ranged from 2.465 to 2.782, Pielou's Evenness index $\left(\mathrm{J}^{\prime}\right)$ from 0.841 to 0.894 , and Berger-Parker index of dominance (d) from 0.122 to 0.243 . Families Libellulidae (Odonata), Coenagrionidae (Odonata) and Gerridae (Hemiptera) had high relative abundance and dominant status. The stream invertebrate grade number-average level (SIGNAL2) score (for family) ranged from 2.316 to 3.174, lying within quadrant 2 of the SIGNAL2 (family) quadrant diagram. This suggested that the water in the area is likely to have high levels of turbidity, salinity, or nutrients, caused naturally or by anthropogenic activities, and the water has low levels of most toxic chemicals.
\end{abstract}

Keywords: Coleoptera, Hemiptera, Odonata, SIGNAL2 (family) score.

Citation: De, K., A. Sarkar, K. Singh, V.P. Uniyal, J.A. Johnson \& S.A. Hussain (2021). Diversity of aquatic insects and biomonitoring of water quality in the upper Ganga River, a Ramsar site: a preliminary assessment. Journal of Threatened Taxa 13(13): 20011-20018. https://doi.org/10.11609/jott.5458.13.13.20011-20018

Copyright: (C) De et al. 2021. Creative Commons Attribution 4.0 International License. JoTT allows unrestricted use, reproduction, and distribution of this article in any medium by providing adequate credit to the author(s) and the source of publication.

Funding: This work was funded by the National Mission for Clean Ganga, Ministry of Jal Shakti, Department of Water Resources, River development and Ganga Rejuvenation, Government of India (Grant No. B-02/2015-16/1259/NMCG-WIIPROPOSAL).

Competing interests: The authors declare no competing interests.

Author details: KRITISH DE worked as project fellow at the Wildlife Institute of India. Presently he is working as Assistant Professor at Sri Sathya Sai University for Human Excellence. His research interests are biodiversity and ecology. ARKOJYOTI SARKAR is working as project fellow. His research interests are biodiversity and ecology. KRITIKA SINGH worked as project intern. Her research interest is monitoring of environmental health. VIRENDRA PRASAD UNIYAL is working as Scientist G. His research interests are ecology and systematics of insects, bioindicators, biodiversity, and ecological monitoring. JEYARAJ ANTONY JOHNSON is working as Scientist E. His research interests are ecology and monitoring of aquatic ecosystems. SYED AINUL HUSSAIN worked as Scientist G. His research interests are aquatic ecology and conservation biology.

Author contributions: KD-conceptualization, field work, formal analysis, writing original draft; AS-field work, writing original draft; KS-field work, writing original draft; VPU-supervision, review and editing the draft; JAJ-supervision, review and editing the draft; SAH-supervision, review and editing the draft, funding acquisition.

Acknowledgements: The authors are thankful to: the National Mission for Clean Ganga, Ministry of Jal Shakti, Department of Water Resources, River Development and Ganga Rejuvenation, Government of India for sponsoring the work under the project 'Biodiversity conservation and Ganga Rejuvenation'; the director and dean, Wildlife Institute of India, for their administrative support for the study; the Environment, Forest and Climate Change Department, Government of Uttar Pradesh for necessary support during fieldwork.

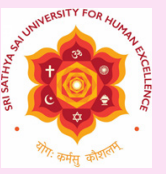




\section{INTRODUCTION}

Freshwater habitats occupy $1 \%$ of the earth's surface (Strayer \& Dudgeon 2010), and in addition to supporting many species freshwater ecosystems provide goods and services of critical importance to human societies. Nevertheless, they are among the most heavily altered ecosystems, with proportional loss of biodiversity (Geist 2011), owing to human activities that have led to widespread habitat degradation, pollution, flow regulation, water extraction, fisheries overexploitation, and alien species introductions (Strayer \& Dudgeon 2010). Alterations of natural flow regimes by manmade dams, land use changes, river impoundments and water abstraction often have profound impacts on lotic communities (Geist 2011). Aquatic insects are an indispensable part of food webs and of nutrient cycling in freshwater ecosystems, and they are essential components of the diets of fish, amphibians and many birds and mammals (Morse 2017). Their abundance and responses to changes in their environment make aquatic insects key indicators for monitoring the effects of human activity on water quality (Adu \& Oyeniyi 2019), and they widely used for freshwater ecosystem monitoring (Souto et al. 2019).

In India, 42 wetlands of international importance (i.e., Ramsar sites) cover 1,081,438 ha according to the Ramsar Sites Information Service (https://rsis.ramsar. org/sites/default /files/rsiswp_search/exports/RamsarSites-annotated-summary-India.pdf?1625598230). Among these wetlands, information on aquatic insect communities and their utility is scant. There are a few studies available on aquatic insect communities of Indian Ramsar sites such as eastern Kolkata wetlands in West Bengal (Sahaet al. 2007), Pong Dam in Himachal Pradesh (Babu et al. 2009), Loktak Lake in Manipur (Takhelmayum \& Gupta 2011, 2015), Deepor beel in Assam (Sharma \& Sharma 2013; Choudhury \& Gupta 2017), and Nalsarovar Bird Sanctuary in Gujarat (Rathod \& Parasharya 2018).

The use of insects as bioindicators is a low-cost strategy for preliminary assessments of the water quality of inland freshwater bodies, as it avoids the use of expensive analytical methods (Pal et al. 2012). The top predators among insects in aquatic ecosystems include aquatic Coleoptera, Hemiptera, and Odonata (Klecka \& Boukal 2012). This study assessed diversity of these groups in the upper Ganga River, a Ramsar site; the goal of using them as indicators of water quality.

\section{MATERIALS AND METHODS}

The study was conducted in an 85-km stretch of the river Ganga from Brijghat to Narora in Uttar Pradesh (Figure 1). This section of the river was declared a Ramsar site in 2005 and is generally characterized by shallow water, although some deep water pools are present inhabited by conservative significant species such as Ganges River Dolphin, Gharial, crocodiles, turtles, otters, 82 species of fish and more than a hundred species of birds. The study was carried out during March 2019. The study area was stratified into 14 sampling sites with a distance of $\sim 5 \mathrm{~km}$ between two sites and insect sampling was done at each site. At each study site, sampling was done between $0930 \mathrm{~h}$ and $1130 \mathrm{~h}$ along the left bank (because of accessibility to the river bank) of the main channel of the river Ganga.

To collect odonates, a $100 \mathrm{~m} \times 20 \mathrm{~m}$ transect (subdivided into 20 segments of $5 \mathrm{~m}$ ) (Juen \& De Marco 2011) was placed at each sampling site parallel to and $\sim 1 \mathrm{~m}$ beside the main river channel. Adult odonates present in each of these segments were captured using insect collection nets (mesh size $60 \mu \mathrm{m}$ ) and released after identification using published pictorial field guides (Andrew et al. 2008; Subramanian 2009; Nair 2011). For Coleoptera and Hemiptera, a circular net (mesh size $60 \mu \mathrm{m}$ ) was dragged in the open water for one minute and continued three times per site (Subramanian \& Sivaramakrishnan 2007). All samples were preserved in $70 \%$ ethanol and brought to the laboratory for further analysis. They were later identified at species level using a stereo zoom microscope with the help of taxonomic literature (Bal \& Basu 1994a,b; Biswas \& Mukhopdhyay 1995; Biswas et al. 1995; Chandra \& Jehamalar 2012).

The aquatic insect data were subjected to Shannon diversity index $\left(\mathrm{H}^{\prime}\right)$, Pielou's evenness index $\left(\mathrm{J}^{\prime}\right)$, and Berger-Parker index of dominance (d) index analysis. The dominant status of the insects was calculated according to Engelmann's scale (1978) in which if relative abundance of a species is up to $1 \%$, it is considered as subrecedent; if between 1.1-3.1\%, recedent; if between $3.2-10 \%$, subdominant; if between $10.1-31.6 \%$, dominant, and if $31.7 \%$ or more then eudominant.

By evaluating comparative performance of several aquatic health indices, Cox et al. (2019) found that the stream invertebrate grade number-average level (SIGNAL2) is the most sensitive index, family richness percentage is the most robust index, family richness and family richness percentage are the best ranked indices for both measures of usability; but Australian River Assessment System (AUSRIVAS OE50), Ephemeroptera 


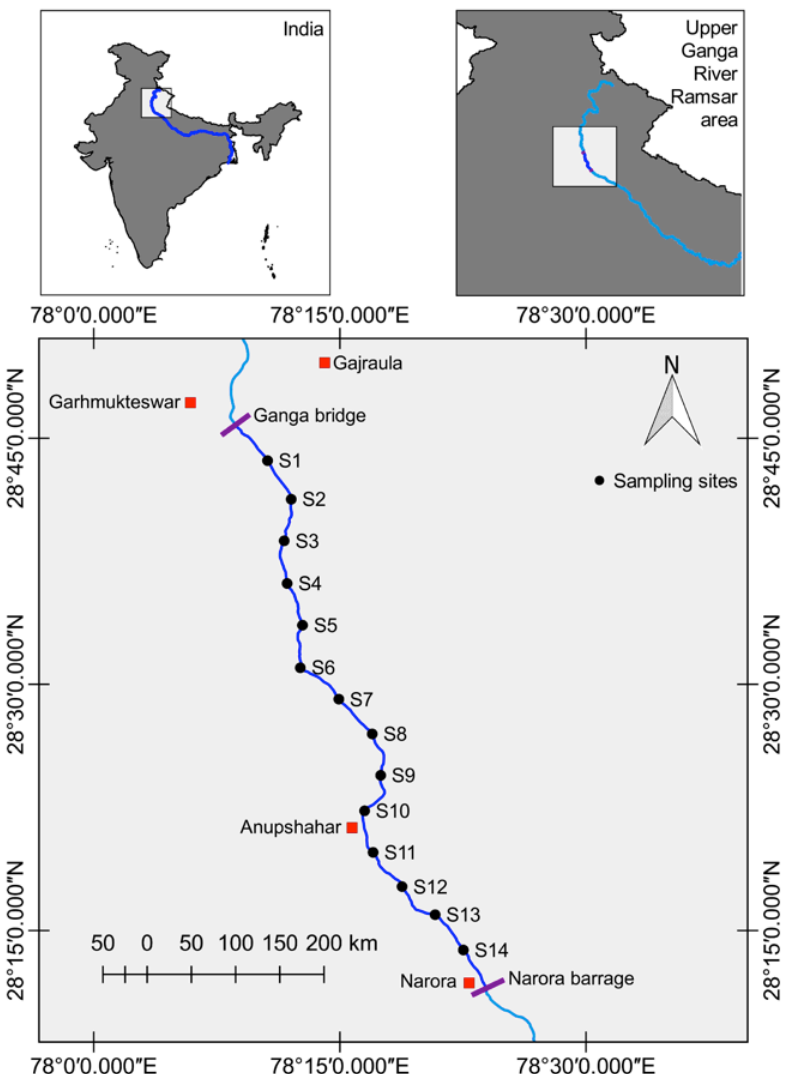

Figure 1. Location of sampling sites (S1-S14) in the Ganga River from Brijghat to Narora, Uttar Pradesh.

Plecoptera \& Trichoptera index (EPT), and Bray-Curtis index $(\mathrm{BCl})$ have poor performance to asses river health condition.

In this study, for the assessment of the bioindicator potential of the insects, SIGNAL2 (family) score was used which is a family-level water pollution index based on the known tolerances of aquatic macro-invertebrate families to various pollutants which has a gradient from 1 to 10 (ranging from a pollution tolerant to a pollution sensitive community) (Chessman et al. 1995). The SIGNAL2 (family) scores were plotted in a quadrant diagram (SIGNAL2 score in the $y$ axis and the numbers of families in the $x$ axis) which includes four quadrants. The first quadrant indicates favourable habitat and chemically dilute waters, the second quadrant indicates high salinity or nutrient levels (may be natural), the third quadrant indicates toxic pollution or harsh physical conditions and the fourth quadrant indicates urban, industrial or agricultural pollution, or downstream effects of dams (Chessman et al. 1995).

All the analyses were performed in the software Past 3 (Hammer et al. 2001) and R 3.5.3 (R Core Team 2019).

\section{RESULTS}

A total of 29 species of aquatic insects were recorded (Table 1), including three species of Coleoptera belonging to two families, four species of Hemiptera belonging to four families, and 22 species of Odonata belonging to three families. Among the odonates, 14 were dragonflies (Suborder Anisoptera) and eight were damselflies (Suborder Zygoptera). Nine species were recorded from all 14 sampling sites: Gerriss pinolae Lethierry \& Severin, 1896; Anisops campbelli Brooks, 1951; Brachythemis contaminata Fabricius, 1793; Diplacodes trivialis Rambur, 1842; Orthetrum sabina Drury, 1770; Trithemis aurora Burmeister, 1839; Ceriagrion coromandelianum Fabricius, 1798; Pseudagrion decorum Rambur, 1842, and Pseudagrion rubriceps Selys, 1876.

The Shannon diversity index $\left(\mathrm{H}^{\prime}\right)$ ranged from 2.465 (at S8) to 2.782 (at S14) (mean= 2.579, SD=0.086); Pielou's evenness index $\left(\mathrm{J}^{\prime}\right)$ was maximum at $\mathrm{S7}\left(\mathrm{J}^{\prime}=0.894\right)$, and Berger-Parker index of dominance (d) ranged from 0.122 (S7) to 0.243 (S11) (mean=0.170, SD=0.037). Variation of Shannon diversity index $\left(\mathrm{H}^{\prime}\right)$, Pielou's evenness index $\left(\mathrm{J}^{\prime}\right)$, and Berger-Parker index of dominance $(\mathrm{d})$ are given in Figure 2.

For families, Gerridae (Hemiptera) was dominant in $>92 \%$ of sampling sites, and Notonectidae (Hemiptera) in $>28 \%$ of sites. Libellulidae (Odonata) was eudominant in $>64 \%$ of sampling sites and dominant in $>35 \%$ of sites, while Coenagrionidae (Odonata) was eudominant in $>71$ $\%$ of the sampling sites, and dominant in $>28 \%$ of sites. Dominance status in different sites is given in Table 2 .

The family richness and the family richness percentage varies from 7 to 9 and 77.77 to $100 \%$, respectively. Highest family richness and family richness percentage was found at S10.

The SIGNAL2 (family) score ranges between 2.316 (S6) and 3.174 (S11) (mean= 2.579, SD=0.086). The family richness, family richness percentage and SIGNAL2 (family) score showed an increasing trend in values from S1 to S14 (Figure 3).

The SIGNAL 2 quadrant diagram plots SIGNAL 2 scores (on $y$ axis) against numbers of aquatic invertebrate families (on $x$ axis). Each diagram has four quadrants which represent different status of water and habitat qualities (Chessman 2003). In the present study, the SIGNAL2 (family) score ranged from 2.316 to 3.174 (Figure 3) and fell within the quadrant 2 (Figure 4). 
Table 1. List of Coleoptera, Hemiptera, and Odonata recorded from across the study area in different sites of Upper Ganga Ramsar site (+ represents presence and - represent absence).

\begin{tabular}{|c|c|c|c|c|c|c|c|c|c|c|c|c|c|c|}
\hline \multirow[t]{2}{*}{ Species } & \multicolumn{14}{|c|}{ Sampling sites } \\
\hline & S1 & $\mathrm{S} 2$ & S3 & S4 & S5 & S6 & S7 & S8 & S9 & S10 & S11 & S12 & $\mathrm{S} 13$ & S14 \\
\hline \multicolumn{15}{|l|}{ Order: Coleoptera } \\
\hline \multicolumn{15}{|l|}{ Family: Dytiscidae (Predaceous Diving Beetle) } \\
\hline 1. Cybister limbatus (Fabricius, 1775) & + & - & - & - & + & + & + & + & - & + & + & + & + & - \\
\hline 2. Eretes sticticus (Linnaeus, 1767) & - & + & - & - & + & - & + & - & + & + & + & + & - & - \\
\hline \multicolumn{15}{|l|}{ Family: Hydrophilidae (Water Scavenger Beetle) } \\
\hline 1. Hydrophilus senegalensis (Percheron 1835) & + & + & + & + & + & + & + & + & + & + & - & + & + & + \\
\hline \multicolumn{15}{|l|}{ Order: Hemiptera } \\
\hline \multicolumn{15}{|l|}{ Family: Belostomatidae (Water Bug) } \\
\hline 1. Diplonychus rusticus (Fabricius, 1781) & - & + & + & + & - & + & - & + & - & + & + & + & - & - \\
\hline \multicolumn{15}{|l|}{ Family: Gerridae (Water Striders) } \\
\hline 1. Gerris spinolae Lethierry \& Severin, 1896 & + & + & + & + & + & + & + & + & + & + & + & + & + & + \\
\hline \multicolumn{15}{|l|}{ Family: Nepidae (Water Scorpion) } \\
\hline 1. Ranatra elongate Fabricius, 1790 & + & - & + & + & + & + & + & - & + & + & + & - & + & + \\
\hline \multicolumn{15}{|l|}{ Family: Notonectidae (Backswimmers) } \\
\hline 1. Anisops campbelli Brooks, 1951 & + & + & + & + & + & + & + & + & + & + & + & + & + & + \\
\hline \multicolumn{15}{|l|}{ Order: Odonata } \\
\hline \multicolumn{15}{|l|}{ Suborder: Anisoptera (Dragonflies) } \\
\hline \multicolumn{15}{|l|}{ Family: Gomphidae } \\
\hline 1. Platygomphus dolabratus Selys, 1854 & - & - & - & - & - & - & - & - & - & - & + & - & - & + \\
\hline $\begin{array}{l}\text { 2. Ictinogomphus rapax Rambur, } 1842 \text { (Indian Common } \\
\text { Clubtail) }\end{array}$ & - & - & - & - & - & - & - & - & - & + & + & + & + & + \\
\hline \multicolumn{15}{|l|}{ Family: Libellulidae Leach, 1815} \\
\hline 1. Acisoma panorpoides Rambur, 1842 (Trumpet Tail) & + & + & + & - & + & + & + & + & + & - & + & + & + & + \\
\hline $\begin{array}{l}\text { 2. Brachydiplax sobrina Rambur, } 1842 \text { (Little Blue Marsh } \\
\text { Hawk) }\end{array}$ & - & - & - & - & - & - & - & - & - & - & - & + & - & - \\
\hline $\begin{array}{l}\text { 3. Brachythemis contaminate Fabricius, } 1793 \text { (Ditch } \\
\text { Jewel) }\end{array}$ & + & + & + & + & + & + & + & + & + & + & + & + & + & + \\
\hline $\begin{array}{l}\text { 4. Crocothemis servilia Drury, } 1770 \text { (Ruddy Marsh } \\
\text { Skimmer) }\end{array}$ & + & - & - & + & + & + & + & + & + & + & + & - & + & + \\
\hline $\begin{array}{l}\text { 5. Diplacodes trivialis Rambur, } 1842 \text { (Blue Ground } \\
\text { Skimmer) }\end{array}$ & + & + & + & + & + & + & + & + & + & + & + & + & + & + \\
\hline 6. Neurothemis tullia (Drury, 1773) (Pied Paddy Skimmer) & - & - & - & - & - & - & - & - & - & - & - & - & - & + \\
\hline 7. Orthetrum sabina Drury, 1770 (Green Marsh Hawk) & + & + & + & + & + & + & + & + & + & + & + & + & + & + \\
\hline 8. Pantala flavescens Fabricius, 1798 (Wandering Glider) & + & - & + & + & + & + & + & - & + & + & - & - & - & + \\
\hline $\begin{array}{l}\text { 9. Rhyothemis variegate Linnaeus, } 1763 \text { (Common } \\
\text { Picturewing) }\end{array}$ & - & - & - & - & + & - & - & - & + & - & - & + & + & + \\
\hline $\begin{array}{l}\text { 10. Tramea basilaris Palisot de Beauvois, } 1805 \text { (Red Marsh } \\
\text { Trotter) }\end{array}$ & - & + & + & + & + & - & - & + & - & - & + & - & + & + \\
\hline $\begin{array}{l}\text { 11. Trithemis aurora Burmeister, } 1839 \text { (Crimson Marsh } \\
\text { Glider) }\end{array}$ & + & + & + & + & + & + & + & + & + & + & + & + & + & + \\
\hline $\begin{array}{l}\text { 12. Urothemis signata Rambur, } 1842 \text { (Greater Crimson } \\
\text { Glider) }\end{array}$ & - & + & - & + & + & + & + & + & + & - & - & - & + & + \\
\hline \multicolumn{15}{|l|}{ Suborder: Zygoptera (Damselflies) } \\
\hline \multicolumn{15}{|l|}{ Family: Coenagrionidae } \\
\hline 1. Agriocnemis lacteolaSelys, 1877 (Milky Dartlet) & + & + & - & - & + & + & + & + & + & + & + & + & + & + \\
\hline 2. Agriocnemis pygmaea Rambur, 1842 (Pygmy Dartlet) & + & - & + & - & - & + & - & + & + & + & + & - & - & + \\
\hline 3. Amphiallagma parvumSelys, 1876 (Azure Dartlet) & + & + & + & + & + & - & + & - & - & - & - & - & + & + \\
\hline $\begin{array}{l}\text { 4. Ceriagrion coromandelianum Fabricius, } 1798 \\
\text { (Coromandel Marsh Dart) }\end{array}$ & + & + & + & + & + & + & + & + & + & + & + & + & + & + \\
\hline 5. Ischnura nursei Morton, 1907 (Pixie Dartlet) & - & - & - & + & - & + & - & - & + & + & - & - & - & + \\
\hline 6. Ischnura rubilioSelys, 1876 (Western Golden Dartlet) & + & + & + & + & - & + & + & - & + & + & + & + & - & + \\
\hline 7. Pseudagrion decorum Rambur, 1842 (Three Lined Dart) & + & + & + & + & + & + & + & + & + & + & + & + & + & + \\
\hline $\begin{array}{l}\text { 8. Pseudagrion rubriceps Selys, } 1876 \text { (Saffron Faced Blue } \\
\text { Dart) }\end{array}$ & + & + & + & + & + & + & + & + & + & + & + & + & + & + \\
\hline
\end{tabular}




\begin{tabular}{|c|c|c|c|c|c|c|c|c|c|c|c|c|c|c|c|c|c|c|c|c|c|c|c|c|}
\hline 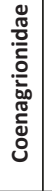 & 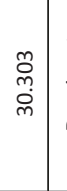 & 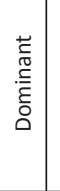 & & 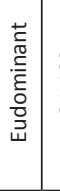 & 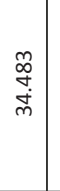 & 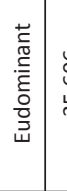 & . & 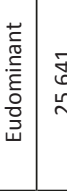 & 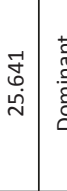 & 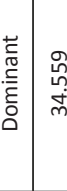 & 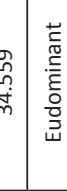 & $\begin{array}{l}\vec{u} \\
\tilde{m} \\
\dot{m} \\
\tilde{m}\end{array}$ & 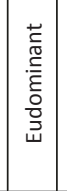 & & 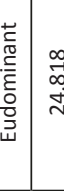 & 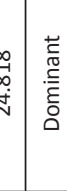 & 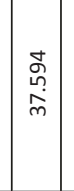 & 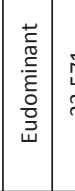 & 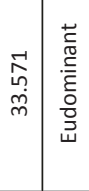 & 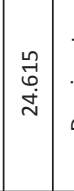 & 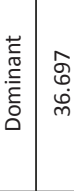 & 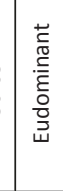 & $\begin{array}{c}\vec{m} \\
\substack{0 \\
\infty \\
m}\end{array}$ & 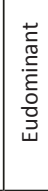 \\
\hline 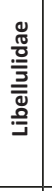 & $\begin{array}{l}\vec{J} \\
\vec{g} \\
\dot{m} \\
.\end{array}$ & 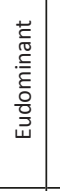 & $\begin{array}{c}\infty \\
\infty \\
\infty \\
\dot{y}\end{array}$ & 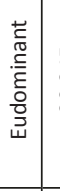 & 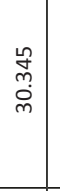 & 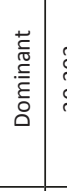 & 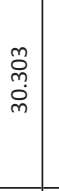 & 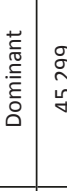 & 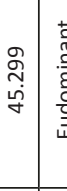 & 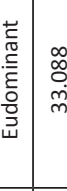 & 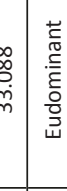 & 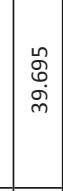 & 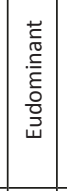 & 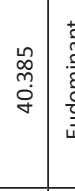 & 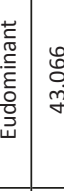 & 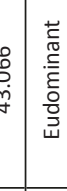 & 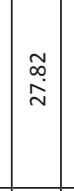 & 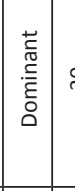 & 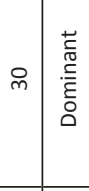 & 点 & & 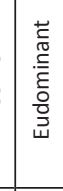 & $\begin{array}{l}\vec{R} \\
\vec{a} \\
\text { i }\end{array}$ & 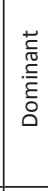 \\
\hline 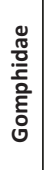 & & & & & & & & & & & & & & & & . & $\begin{array}{l}\tilde{N} \\
\hat{0} \\
0\end{array}$ & 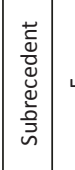 & 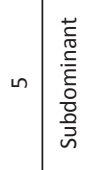 & 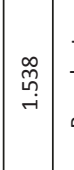 & & 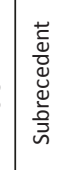 & $\begin{array}{l}\tilde{n} \\
\stackrel{\leftrightarrow}{\text { r. }}\end{array}$ & 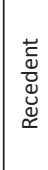 \\
\hline 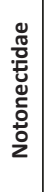 & 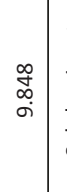 & 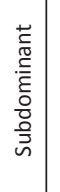 & 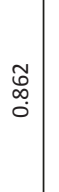 & 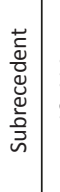 & 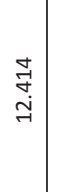 & 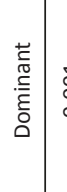 & $\begin{array}{l}\vec{\sigma} \\
\dot{\sigma} \\
\sigma\end{array}$ & 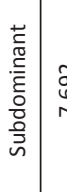 & 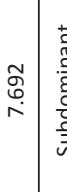 & 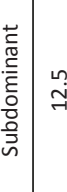 & 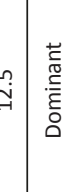 & 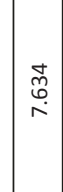 & 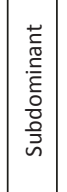 & 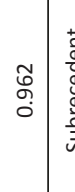 & 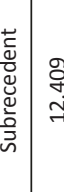 & 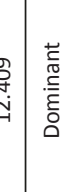 & 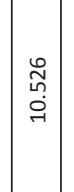 & 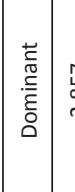 & 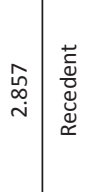 & 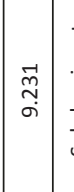 & & 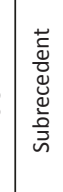 & $\begin{array}{l}\stackrel{J}{\sigma} \\
\sigma \\
\sigma\end{array}$ & 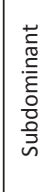 \\
\hline 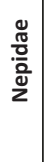 & 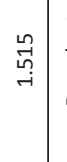 & 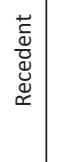 & & & $\begin{array}{l}0 \\
: \\
0 \\
0\end{array}$ & 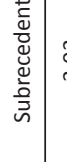 & $\stackrel{m}{\dot{m}}$ & 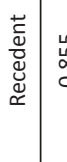 & 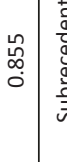 & 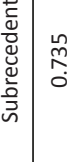 & 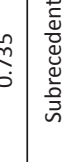 & $\underset{\text { ন }}{\text { \} }} &{\text { 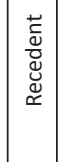 }} &{ } &{\stackrel{9}{\pi}} &{\text { 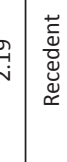 }} &{\stackrel{\stackrel{D}{d}}{i}} &{\text { 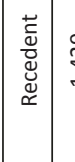 }} &{\text { 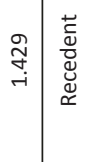 }} &{\text { o }} &{\text { 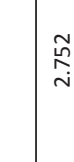 }} &{\text { 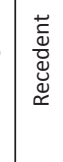 }} &{\text { 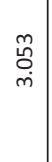 }} &{\text { 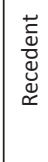 }} \\
{\hline \text { 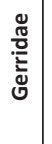 }} &{\begin{array}{l}\underset{N}{\tilde{N}} \\
\underset{N}{1}\end{array}} &{\text { 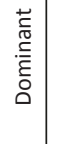 }} &{\text { 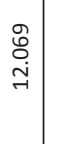 }} &{\text { 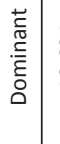 }} &{\text { 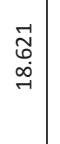 }} &{\text { 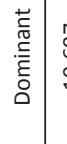 }} &{\begin{array}{l}\hat{\theta} \\
\stackrel{\sigma}{a} \\
\stackrel{a}{2}\end{array}} &{\text { 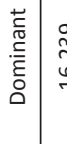 }} &{\text { 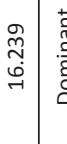 }} &{\text { 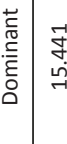 }} &{\text { 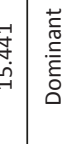 }} &{\stackrel{q}{\exists}} &{\text { 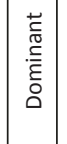 }} &{\text { 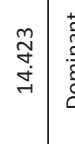 }} &{\text { 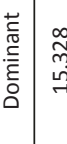 }} &{ } &{\text { 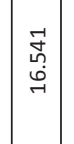 }} &{\text { 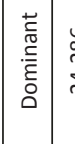 }} &{\text { 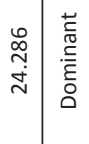 }} &{\text { 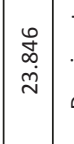 }} &{ } &{\text { 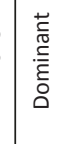 }} &{\text { 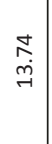 }} &{\text { 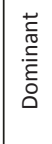 }} \\
{\hline \text { 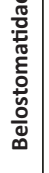 }} &{ } &{ } &{\text { 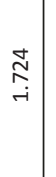 }} &{\text { 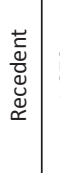 }} &{\text { 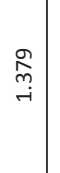 }} &{\text { 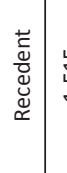 }} &{\text { 怘 }} &{\text { 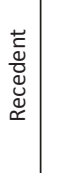 }} &{ } &{\text { 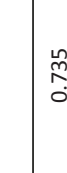 }} &{\text { 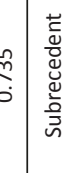 }} &{ } &{ } &{\text { 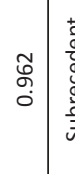 }} &{\text { 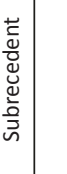 }} &{ } &{\begin{array}{l}\tilde{n} \\
\hat{0}\end{array}} &{\text { 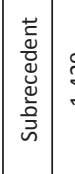 }} &{\text { 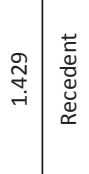 }} &{\text { 畺 }} &{ } &{ } &{ } &{ } \\
{\hline \text { 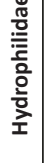 }} &{\text { 㞻 }} &{\text { 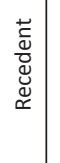 }} &{\text { 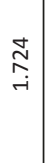 }} &{\text { 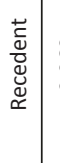 }} &{\begin{array}{l}\stackrel{\circ}{:} \\
\stackrel{i}{ }\end{array}} &{\text { 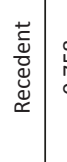 }} &{\text { 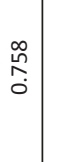 }} &{\text { 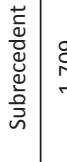 }} &{\text { 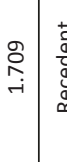 }} &{\text { 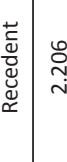 }} &{\text { 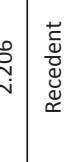 }} &{\text { ָָ }} &{\text { 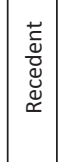 }} &{\text { 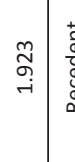 }} &{\text { 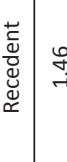 }} &{\text { 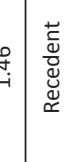 }} &{\text { 芯 }} &{\text { 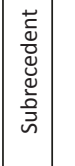 }} &{ } &{\text { 罵 }} &{ } &{\text { 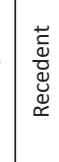 }} &{\begin{array}{l}\stackrel{\hat{n}}{\mathrm{H}} \\
\mathrm{H}\end{array}} &{\text { 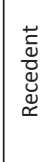 }} \\
{\hline \text { 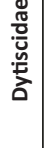 }} &{\text { 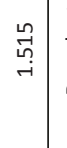 }} &{\text { 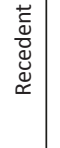 }} &{\underset{i}{\stackrel{\sim}{\sim}}} &{\text { 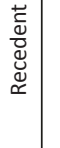 }} &{ } &{ } &{ } &{ } &{\text { 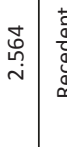 }} &{\text { 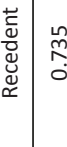 }} &{\text { 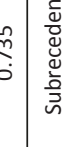 }} &{\text { 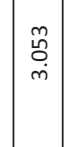 }} &{\text { 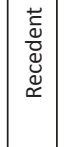 }} &{\text { 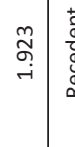 }} &{\text { 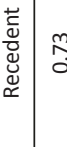 }} &{\text { 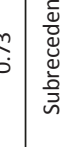 }} &{\begin{array}{l}\infty \\
\stackrel{\infty}{\infty} \\
\dot{m}\end{array}} &{\text { 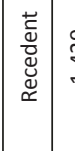 }} &{\text { 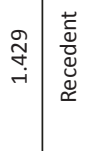 }} &{\text { 离 }} &{ } &{\text { 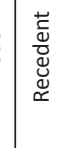 }} &{ } &{ } \\
{\hline} &{\text { 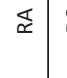 }} &{\check{a}} &{\text { 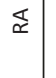 }} &{\text { a }} &{\text { 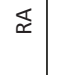 }} &{\ddot{a}} &{\text { 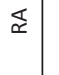 }} &{\tilde{a}} &{\overleftarrow{\Phi}} &{\begin{array}{l|l}\text { 口. } & ₫\end{array}} &{\begin{array}{l}\vec{x} \\
\vec{x}\end{array}} &{\nsubseteq} &{\text { 号 }} &{\begin{array}{l|l}\mathbb{x} \\
\end{array}} &{\text { దิ }} &{\frac{\pi}{2}} &{\text { 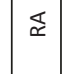 }} &{\text { 品 }} &{\begin{array}{l|l} & \check{a}\end{array}} &{\overleftarrow{\Phi}} &{\text { 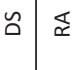 }} &{\text { ฉ }} &{\overleftarrow{\nwarrow}} &{\text { 品 }} \\
{\hline} &{\vec{n}} &{5} &{\tilde{\omega}} &{ } &{\text { 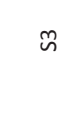 }} &{ } &{\dot{H}} &{ } &{\tilde{n}} &{ } &{\stackrel{\circ}{n}} &{ } &{\hat{n}} &{\stackrel{\infty}{n}} &{ } &{\text { ळr }} &{\text { ज्ञ }} &{\text { 宛 }} &{\vec{H}} &{\tilde{n}} &{ } &{\stackrel{m}{\omega}} &{\bar{j}} &{\vec{s}} \\
$\hline
\end{tabular}



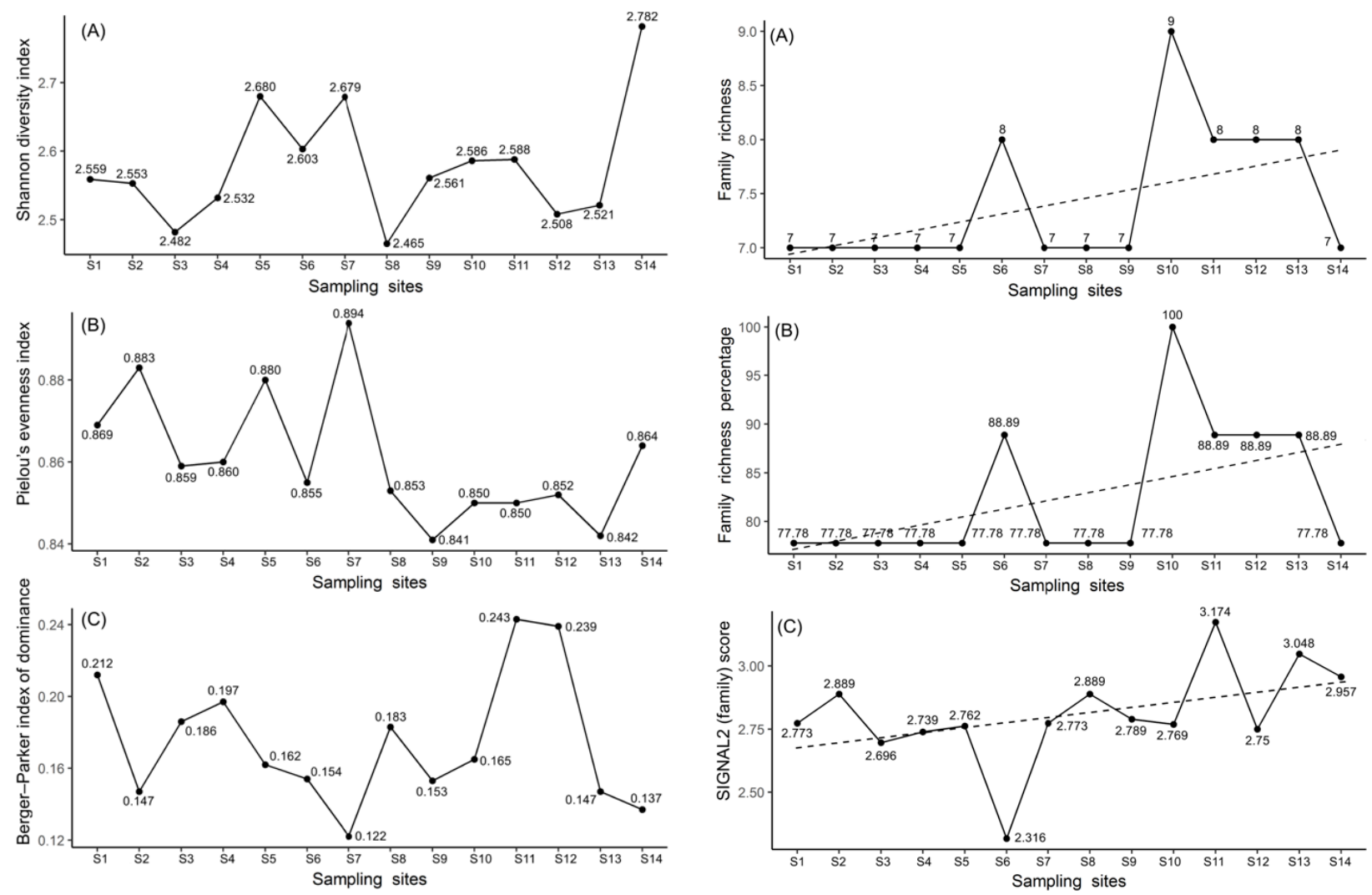

Figure 2. Variation of Shannon diversity index, Pielou's evenness index, and Berger-Parker index of dominance in different sites (S1S14) of the upper Ganga River Ramsar site.

Figure 3. Variation of Family richness, Family richness percentage and SIGNAL2 (family) score in different sites (S1-S14) of the upper Ganga River Ramsar site. The linear trend lines are showing increase of values towards the Narora barrage (at S14).

\section{DISCUSSION}

Insects have the ability to move from unfavourable habitats to favourable ones. If a habitat becomes polluted or altered, tolerant species will thrive and sensitive ones will move to a more suitable habitat (Medina et al. 2007). Thus habitat alternation, either by natural process or by anthropogenic impacts, can shape invertebrate communities. Aquatic macroinvertebrates constitute important components of their ecosystems, and they exhibit differential tolerances to changes in environmental conditions (Adu \& Oyeniyi 2019). In the present study, three species of Coleoptera from two families, four species of Hemiptera from four families, and 22 species of Odonata from three families were recorded. The coleopterans included predaceous diving beetles (family Dytiscidae) and water scavenger beetles (family Hydrophilidae). The hemipteran group included water bugs (family Belostomatidae), water striders (family Gerridae), water scorpions (family Nepidae) and backswimmers (family Notonectidae), and the odonates included dragonflies and damselflies.

In the present study Shannon diversity index $\left(\mathrm{H}^{\prime}\right)$,

Pielou's Evenness index $\left(\mathrm{J}^{\prime}\right)$ and Berger-Parker index of dominance (d) did not differ much between study sites, probably because of uniform geomorphological features of the area, as geomorphological heterogeneity plays a major role in determining species richness (Nichols et al. 1998). Libellulidae, Coenagrionidae, and Gerridae had high relative abundance and dominant status, probably because of their ability to tolerate a wide range of environmental factors (Spence 1983; Chang et al. 2014).

The SIGNAL 2 result suggested that the water of the study area was likely to have higher levels of turbidity, salinity or nutrients, which was perhaps caused either naturally, because of local geology and soil types, or as a result of human activities and physical conditions. Toxic chemicals were not present in large amounts (Chessman 2003).

The family richness, family richness percentage and SIGNAL2 (family) score showed an increase in values towards the Narora barrage, probably because of the increase in water quantity (as the barrage stores more water) which directly affects the physiochemical 


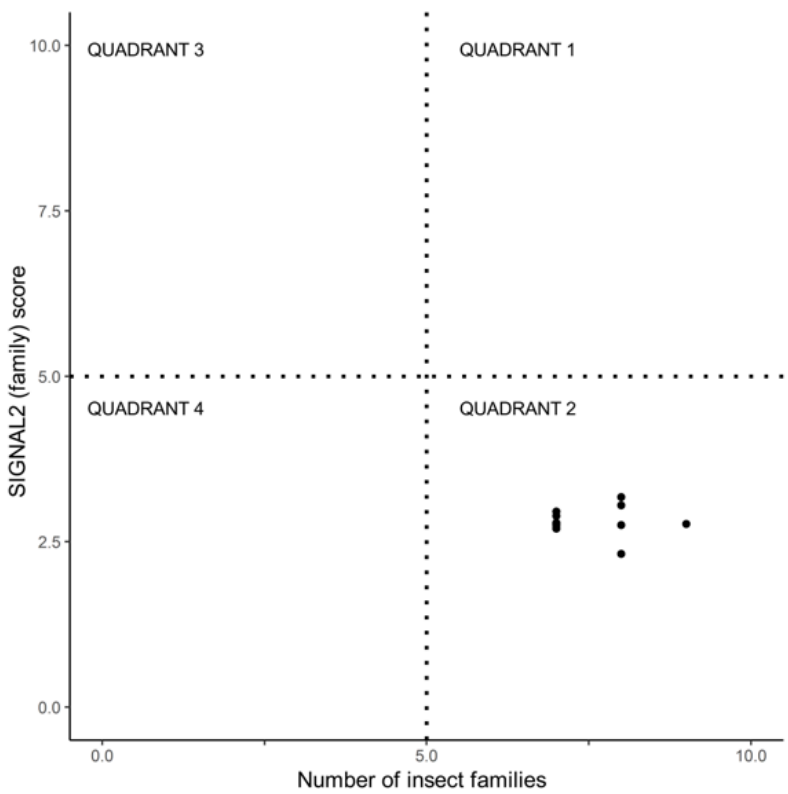

Figure 4. The quadrant diagram for the family version of SIGNAL 2 for the sampling sites.

properties of the water and habitat structures.

The upper Ganga Ramsar site is facing stress from anthropogenic pressure (Kuniyal 2013; Pandey \& Sharma 2013). The study stretch between the Brijghat and Narora, the Ganga is characterized by the presence of agricultural lands and numerous ghats (steps leading down to the river) with religious and tourism importance on both the banks. Local people use the river bank and water for bathing, cremation and other religious activities. Activities like cattle grazing and fishing occur throughout the year. As a result, the river is exposed to various threats like waste discharge, sewage disposal, agricultural runoff, fishing, and river bank erosion.

While there is some regional information, knowledge remains limited concerning the natural ranges and ecology of species found in the Ganga (Nautiyal et al. 2014). Long-term seasonal monitoring of the physiochemical properties of the water, coupled with assessment of faunal and floral diversity as well as socioeconomic factors influencing the conditions of the area is recommended in order to arrive at better management strategies.

\section{REFERENCES}

Adu, B.W. \& E.A. Oyeniyi (2019). Water quality parameters and aquatic insect diversity in Aahoo stream, southwestern Nigeria. The Journal of Basic and Applied Zoology 80(1): 15. https://doi.org/10.1186/ s41936-019-0085-3

Andrew, R.J., K.A. Subramaniam \& A.D. Tiple (2008). Common
Odonates of Central India. E-book for The $18^{\text {th }}$ International Symposium of Odonatology. Hislop College, Nagpur, 50pp.

Babu, R., H.S. Mehta \& S. Kamal (2009). Insecta: Odonata,pp. 13-19. In: Wetland Ecosystem Series 12: Faunal Diversity of Pong Dam and its Catchment Area (District Kangra, Himachal Pradesh). Zoological Survey of India; Kolkata, 138pp.

Bal, A. \& R.C. Basu (1994a). Insecta: Hemiptera: Belostomatidae, Nepidae, Notonectidae And Pleidae. State Fauna Series 3: Fauna of West Bengal, Part 5 (Insecta: Hemiptera). Zoological Survey of India, Calcutta, 558pp.

Bal, A. \& R.C. Basu (1994b). Insecta: Hemiptera: Mesoveliidae, Hydrometridae, Veliidae and Gerrida. State Fauna Series 3: Fauna of West Bengal, Part 5 (Insecta: Hemiptera). Zoological Survey of India, Calcutta, 558pp.

Biswas B. \& P. Mukhopdhyay (1995). Insecta: Coleoptera: Hydrophilidae. State Fauna Series 1: Fauna of West Bengal, Part 6A. Zoological Survey of India, Calcutta, 447pp.

Biswas, S., P. Mukhopadhyay \& S.K. Saha (1995). Insecta: Coleoptera: Adephaga: Fam. Dytiscidae. State Fauna Series 1: Fauna of West Bengal, Part 6A. Zoological Survey of India, Calcutta, 447pp.

Chandra, K. \& E.E. Jehamalar (2012). Morphological differences in three species of the genus Diplonychus (Hemiptera: Belostomatidae) known from India. Records of the Zoological Survey of India 112(2): 91-99.

Chang Y.-H., C.-R. Ku \& H.-L. Lu (2014). Effects of aquatic ecological indicators of sustainable green energy landscape facilities. Ecological Engineering 71: 144-153. https://doi.org/10.1016/j. ecoleng.2014.07.051

Chessman, B. (2003). SIGNAL 2 - A Scoring System for Macroinvertebrate ('Water Bugs') in Australian Rivers, Monitoring River Heath Initiative Technical Report no 31. Canberra: Commonwealth of Australia, 32pp.

Choudhury, D. \& S. Gupta (2017). Impact of waste dump on surface water quality and aquatic insect diversity of DeeporBeel (Ramsar site), Assam, North-east India. Environmental Monitoring and Assessment 189(11): 540. https://doi.org/10.1007/s10661-0176233-7

Cox, B., S. Oeding \& K. Taffs (2019). A comparison of macroinvertebratebased indices for biological assessment of river health: a case example from the sub-tropical Richmond River Catchment in northeast New South Wales, Australia. Ecological Indicators 106: 105479. https://doi.org/10.1016/j.ecolind.2019.105479

Engelmann, H.D. (1978). Zurdominanzklassifizierung von Bodenarthropoden. Pedobiologia 18: 378-380.

Geist, J. (2011). Integrative freshwater ecology and biodiversity conservation. Ecological Indicators 11(6): 1507-1516. https://doi. org/10.1016/j.ecolind.2011.04.002

Hammer, Ø., D.A.T. Harper \& P.D. Ryan (2001). PAST: Paleontological statistics software package for education and data analysis. Palaeontologia Electronica 4(1): 1-9.

Juen, L. \& P. De Marco, Jr. (2011). Odonate biodiversity in terra-firme forest streamlets in Central Amazonia: on the relative effects of neutral and niche drivers at small geographical extents. Insect Conservation and Diversity 4(4): 265-274. https://doi.org/10.1111/ j.1752-4598.2010.00130.x

Klecka, J. \& D.S. Boukal (2012). Who eats whom in a pool? A comparative study of prey selectivity by predatory aquatic insects. PLoS One 7(6): e37741. https://doi.org/10.1371/journal. pone.0037741

Kuniyal, C.P. (2013). Siddhwari sacred grove in Upper Ganga Ramsar site of Uttar Pradesh. Current Science 105(8): 1039-1040.

Medina, M.H., J.A. Correa \& C. Barata (2007). Micro-evolution due to pollution: Possible consequences for ecosystem responses to toxic stress. Chemosphere 67(11): 2105-2114. https://doi.org/10.1016/j. chemosphere.2006.12.024

Morse, J.C. (2017). Biodiversity of aquatic insects,pp. 205-227 In: Foottit R.G. \& P.H. Adler (eds.). Insect Biodiversity: Science and Society, Vol 1, $2^{\text {nd }}$ Edition. John Wiley and Sons Ltd, New Jersey, 904pp. 
Nair, M.V. (2011). Dragonflies and Damselflies of Orissa and Eastern India. Bhubaneswar: Wildlife Organisation, Forest and Environment Department, Government of Orissa, 252pp.

Nautiyal, P., J. Verma \& A.S. Mishra (2014). Distribution of major flora and faunal biodiversity in the Mountain and Upper Gangetic Plains section of the river Ganga: Diatoms, macroinvertebrates and fish,pp. 75-119. In: Shanghi, R. (ed.). Our National River Ganga: Lifeline of Millions. Springer International Publishing, 415pp.

Nichols, W.F., K.T Killingbeck \& P.V. August (1998). The influence of geomorphological heterogeneity on biodiversity II. A landscape perspective. Conservation Biology 12(2): 371-379. https://doi. org/10.1111/j.1523-1739.1998.96237.x

Pal, A., D.C. Sinha \& N. Rastogi (2012). Gerriss pinolae Lethierry and Severin (hemiptera: Gerridae) and Brachydeuteralongipes Hende (Diptera: Ephydridae): two effective insect bioindicators to monito pollution in some tropical freshwater ponds under anthropogenic stress. Psyche: A Journal of Entomology 2012: 818490. https://doi. org/10.1155/2012/818490

Pandey, A. \& G.P. Sharma (2013). Mandu sacred grove in Upper Ganga Ramsar site, Uttar Pradesh. Current Science 104(4): 409-410.

R Core Team (2019). R: A language and environment for statistica computing. R Foundation for Statistical Computing, Vienna, Austria. URL https://www.R-project.org/

Rathod, D.M. \& B.M. Parasharya (2018). Odonate diversity of Nalsarovar Bird Sanctuary-a Ramsar site in Gujarat, India. Journal of Threatened Taxa 10(8): 12117-12122. https://doi.org/10.11609/ jott.4017.10.8.12117-12122

Saha, N., G. Aditya, A. Bal \& G.K. Saha (2007). Comparative study of functional response of common Hemipteran bugs of East Calcutta wetlands, India. International Review of Hydrobiology 92(3): 242257. https://doi.org/10.1002/iroh.200610939
Sharma, S. \& B.K. Sharma (2013). Faunal diversity of aquatic invertebrates of Deepor Beel (a Ramsar site), Assam, northeast India. In: Wetland Ecosystem Series, 17. Zoological Survey of India, Kolkata, 227pp.

Souto, R.D.M.G., J.J. Corbi \& G.B. Jacobucci (2019). Aquatic insects as bioindicators of heavy metals in sediments in Cerrado streams. Limnetica 38(2): 575-586. https://doi.org/10.23818/limn.38.33

Spence, J.R. (1983). Pattern and process in co-existence of waterstriders (Heteroptera: Gerridae). The Journal of Animal Ecology 52(2): 497-511.

Strayer D.L. \& D. Dudgeon (2010). Freshwater biodiversity conservation: recent progress and future challenges. Journal of the North American Benthological Society 29(1): 344-358. https://doi. org/10.1899/08-171.1

Subramanian, K.A. \& K.G. Sivaramakrishnan (2007). Aquatic Insects for Biomonitoring Freshwater Ecosystems - A Methodology Manual. Asoka Trust for Research in Ecology and Environment (ATREE), Bangalore, 31pp.

Subramanian, K.A. (2009). Dragonflies of India - A Field Guide. Vigyan Prasar, Department of Science and Technology, Noida, 168pp.

Takhelmayum, K. \& S. Gupta (2011). Distribution of aquatic insects in phumdis (floating island) of Loktak Lake, Manipur, northeastern India. Journal of Threatened Taxa 3(6): 1856-1861. https://doi. org/10.11609/JoTT.o2526.1856-61

Takhelmayum, K. \& S. Gupta (2015). Aquatic insect diversity of a protected area, KeibulLamjao National Park in Manipur, North East India. Journal of Asia Pacific Entomology 18(2): 335-341. https:// doi.org/10.1016/j.aspen.2015.04.002 
Dr. John Noyes, Natural History Museum, London, UK

Dr. Albert G. Orr, Griffith University, Nathan, Australia

Dr. Sameer Padhye, Katholieke Universiteit Leuven, Belgium

Dr. Nancy van der Poorten, Toronto, Canada

Dr. Kareen Schnabel, NIWA, Wellington, New Zealand

Dr. R.M. Sharma, (Retd.) Scientist, Zoological Survey of India, Pune, India

Dr. Manju Siliwal, WILD, Coimbatore, Tamil Nadu, India

Dr. G.P. Sinha, Botanical Survey of India, Allahabad, India

Dr. K.A. Subramanian, Zoological Survey of India, New Alipore, Kolkata, India

Dr. P.M. Sureshan, Zoological Survey of India, Kozhikode, Kerala, India

Dr. R. Varatharajan, Manipur University, Imphal, Manipur, India

Dr. Eduard Vives, Museu de Ciències Naturals de Barcelona, Terrassa, Spain

Dr. James Young, Hong Kong Lepidopterists' Society, Hong Kong

Dr. R. Sundararaj, Institute of Wood Science \& Technology, Bengaluru, India

Dr. M. Nithyanandan, Environmental Department, La Ala Al Kuwait Real Estate. Co. K.S.C.,

Kuwait

Dr. Himender Bharti, Punjabi University, Punjab, India

Mr. Purnendu Roy, London, UK

Dr. Saito Motoki, The Butterfly Society of Japan, Tokyo, Japan

Dr. Sanjay Sondhi, TITLI TRUST, Kalpavriksh, Dehradun, India

Dr. Nguyen Thi Phuong Lien, Vietnam Academy of Science and Technology, Hanoi, Vietnam

Dr. Nitin Kulkarni, Tropical Research Institute, Jabalpur, India

Dr. Robin Wen Jiang Ngiam, National Parks Board, Singapore

Dr. Lional Monod, Natural History Museum of Geneva, Genève, Switzerland.

Dr. Asheesh Shivam, Nehru Gram Bharti University, Allahabad, India

Dr. Rosana Moreira da Rocha, Universidade Federal do Paraná, Curitiba, Brasi

Dr. Kurt R. Arnold, North Dakota State University, Saxony, Germany

Dr. James M. Carpenter, American Museum of Natural History, New York, USA

Dr. David M. Claborn, Missouri State University, Springfield, USA

Dr. Kareen Schnabel, Marine Biologist, Wellington, New Zealand

Dr. Amazonas Chagas Júnior, Universidade Federal de Mato Grosso, Cuiabá, Brasil

Mr. Monsoon Jyoti Gogoi, Assam University, Silchar, Assam, India

Dr. Heo Chong Chin, Universiti Teknologi MARA (UiTM), Selangor, Malaysia

Dr. R.J. Shiel, University of Adelaide, SA 5005, Australia

Dr. Siddharth Kulkarni, The George Washington University, Washington, USA

Dr. Priyadarsanan Dharma Rajan, ATREE, Bengaluru, India

Dr. Phil Alderslade, CSIRO Marine And Atmospheric Research, Hobart, Australia

Dr. John E.N. Veron, Coral Reef Research, Townsville, Australia

Dr. Daniel Whitmore, State Museum of Natural History Stuttgart, Rosenstein, Germany.

Dr. Yu-Feng Hsu, National Taiwan Normal University, Taipei City, Taiwan

Dr. Keith V. Wolfe, Antioch, California, USA

Dr. Siddharth Kulkarni, The Hormiga Lab, The George Washington University, Washington,

D.C., USA

Dr. Tomas Ditrich, Faculty of Education, University of South Bohemia in Ceske

Budejovice, Czech Republic

Dr. Mihaly Foldvari, Natural History Museum, University of Oslo, Norway

Dr. V.P. Uniyal, Wildlife Institute of India, Dehradun, Uttarakhand 248001, India

Dr. John T.D. Caleb, Zoological Survey of India, Kolkata, West Bengal, India

Dr. Priyadarsanan Dharma Rajan, Ashoka Trust for Research in Ecology and the Environment

(ATREE), Royal Enclave, Bangalore, Karnataka, India

\section{Fishes}

Dr. Neelesh Dahanukar, IISER, Pune, Maharashtra, India

Dr. Topiltzin Contreras MacBeath, Universidad Autónoma del estado de Morelos, México

Dr. Heok Hee Ng, National University of Singapore, Science Drive, Singapore

Dr. Rajeev Raghavan, St. Albert's College, Kochi, Kerala, India

Dr. Robert D. Sluka, Chiltern Gateway Project, A Rocha UK, Southall, Middlesex, UK

Dr. E. Vivekanandan, Central Marine Fisheries Research Institute, Chennai, India

Dr. Davor Zanella, University of Zagreb, Zagreb, Croatia

Dr. A. Biju Kumar, University of Kerala, Thiruvananthapuram, Kerala, India

Dr. Akhilesh K.V., ICAR-Central Marine Fisheries Research Institute, Mumbai Research

Centre, Mumbai, Maharashtra, India

Dr. J.A. Johnson, Wildlife Institute of India, Dehradun, Uttarakhand, India

Amphibians

Dr. Sushil K. Dutta, Indian Institute of Science, Bengaluru, Karnataka, India

Dr. Annemarie Ohler, Muséum national d'Histoire naturelle, Paris, France

\section{Reptiles}

Dr. Gernot Vogel, Heidelberg, Germany

Dr. Raju Vyas, Vadodara, Gujarat, India

Dr. Pritpal S. Soorae, Environment Agency, Abu Dubai, UAE.

Prof. Dr. Wayne J. Fuller, Near East University, Mersin, Turkey

Prof. Chandrashekher U. Rivonker, Goa University, Taleigao Plateau, Goa. India

Dr. S.R. Ganesh, Chennai Snake Park, Chennai, Tamil Nadu, India

Dr. Himansu Sekhar Das, Terrestrial \& Marine Biodiversity, Abu Dhabi, UAE
Birds

Dr. Hem Sagar Baral, Charles Sturt University, NSW Australia

Dr. Chris Bowden, Royal Society for the Protection of Birds, Sandy, UK

Dr. Priya Davidar, Pondicherry University, Kalapet, Puducherry, India

Dr. J.W. Duckworth, IUCN SSC, Bath, UK

Dr. Rajah Jayapal, SACON, Coimbatore, Tamil Nadu, India

Dr. Rajiv S. Kalsi, M.L.N. College, Yamuna Nagar, Haryana, India

Dr. V. Santharam, Rishi Valley Education Centre, Chittoor Dt., Andhra Pradesh, India

Dr. S. Balachandran, Bombay Natural History Society, Mumbai, India

Mr. J. Praveen, Bengaluru, India

Dr. C. Srinivasulu, Osmania University, Hyderabad, India

Dr. K.S. Gopi Sundar, International Crane Foundation, Baraboo, USA

Dr. Gombobaatar Sundev, Professor of Ornithology, Ulaanbaatar, Mongolia

Prof. Reuven Yosef, International Birding \& Research Centre, Eilat, Israel

Dr. Taej Mundkur, Wetlands International, Wageningen, The Netherlands

Dr. Carol Inskipp, Bishop Auckland Co., Durham, UK

Dr. Tim Inskipp, Bishop Auckland Co, Durham, UK

Dr. V. Gokula, National College, Tiruchirappalli, Tamil Nadu, India

Dr. Arkady Lelej, Russian Academy of Sciences, Vladivostok, Russia

Dr. Simon Dowell, Science Director, Chester Zoo, UK

Dr. Mário Gabriel Santiago dos Santos, Universidade de Trás-os-Montes e Alto Douro,

Quinta de Prados, Vila Real, Portugal

Dr. Grant Connette, Smithsonian Institution, Royal, VA, USA

Dr. M. Zafar-ul Islam, Prince Saud Al Faisal Wildlife Research Center, Taif, Saudi Arabia

Mammals

Dr. Giovanni Amori, CNR - Institute of Ecosystem Studies, Rome, Italy

Dr. Anwaruddin Chowdhury, Guwahati, India

Dr. David Mallon, Zoological Society of London, UK

Dr. Shomita Mukherjee, SACON, Coimbatore, Tamil Nadu, India

Dr. Angie Appel, Wild Cat Network, Germany

Dr. P.O. Nameer, Kerala Agricultural University, Thrissur, Kerala, India

Dr. Ian Redmond, UNEP Convention on Migratory Species, Lansdown, UK

Dr. Heidi S. Riddle, Riddle's Elephant and Wildlife Sanctuary, Arkansas, USA

Dr. Karin Schwartz, George Mason University, Fairfax, Virginia.

Dr. Lala A.K. Singh, Bhubaneswar, Orissa, India

Dr. Mewa Singh, Mysore University, Mysore, India

Dr. Paul Racey, University of Exeter, Devon, UK

Dr. Honnavalli N. Kumara, SACON, Anaikatty P.O., Coimbatore, Tamil Nadu, India

Dr. Nishith Dharaiya, HNG University, Patan, Gujarat, India

Dr. Spartaco Gippoliti, Socio Onorario Società Italiana per la Storia della Fauna "Giuseppe

Altobello", Rome, Italy

Dr. Justus Joshua, Green Future Foundation, Tiruchirapalli, Tamil Nadu, India

Dr. H. Raghuram, The American College, Madurai, Tamil Nadu, India

Dr. Paul Bates, Harison Institute, Kent, UK

Dr. Jim Sanderson, Small Wild Cat Conservation Foundation, Hartford, USA

Dr. Dan Challender, University of Kent, Canterbury, UK

Dr. David Mallon, Manchester Metropolitan University, Derbyshire, UK

Dr. Brian L. Cypher, California State University-Stanislaus, Bakersfield, CA

Dr. S.S. Talmale, Zoological Survey of India, Pune, Maharashtra, India

Prof. Karan Bahadur Shah, Budhanilakantha Municipality, Kathmandu, Nepal

Dr. Susan Cheyne, Borneo Nature Foundation International, Palangkaraja, Indonesia

Dr. Hemanta Kafley, Wildlife Sciences, Tarleton State University, Texas, USA

\section{Other Disciplines}

Dr. Aniruddha Belsare, Columbia MO 65203, USA (Veterinary)

Dr. Mandar S. Paingankar, University of Pune, Pune, Maharashtra, India (Molecular)

Dr. Jack Tordoff, Critical Ecosystem Partnership Fund, Arlington, USA (Communities)

Dr. Ulrike Streicher, University of Oregon, Eugene, USA (Veterinary)

Dr. Hari Balasubramanian, EcoAdvisors, Nova Scotia, Canada (Communities)

Dr. Rayanna Hellem Santos Bezerra, Universidade Federal de Sergipe, São Cristóvão, Brazil

Dr. Jamie R. Wood, Landcare Research, Canterbury, New Zealand

Dr. Wendy Collinson-Jonker, Endangered Wildlife Trust, Gauteng, South Africa

Dr. Rajeshkumar G. Jani, Anand Agricultural University, Anand, Gujarat, India

Dr. O.N. Tiwari, Senior Scientist, ICAR-Indian Agricultural Research Institute (IARI), New

Delhi, India

Dr. L.D. Singla, Guru Angad Dev Veterinary and Animal Sciences University, Ludhiana, India

Dr. Rupika S. Rajakaruna, University of Peradeniya, Peradeniya, Sri Lanka

Dr. Bahar Baviskar, Wild-CER, Nagpur, Maharashtra 440013, India

Reviewers 2018-2020

Due to pausity of space, the list of reviewers for $2018-2020$ is available online.

The opinions expressed by the authors do not reflect the views of the Journal of Threatened Taxa, Wildlife Information Liaison Development Society, Zoo Outreach Organization, or any of the partners. The journal, the publisher, the host, and the partners are not responsible for the accuracy of the political boundaries shown in the maps by the authors.

Journal of Threatened Taxa is indexed/abstracted in Bibliography of Systematic Mycology, Biological Abstracts, BIOSIS Previews, CAB Abstracts, EBSCO, Google Scholar, Index Copernicus, Index Fungorum, JournalSeek, National Academy of Agricultural Sciences, NewJour, OCLC WorldCat, SCOPUS, Stanford University Libraries, Virtual Library of Biology, Zoological Records.

NAAS rating (India) 5.64
Print copies of the Journal are available at cost. Write to:

The Managing Editor, JoTT,

c/o Wildlife Information Liaison Development Society,

No. 12, Thiruvannamalai Nagar, Saravanampatti - Kalapatti Road,

Saravanampatti, Coimbatore, Tamil Nadu 641035, India

ravi@threatenedtaxa.org 


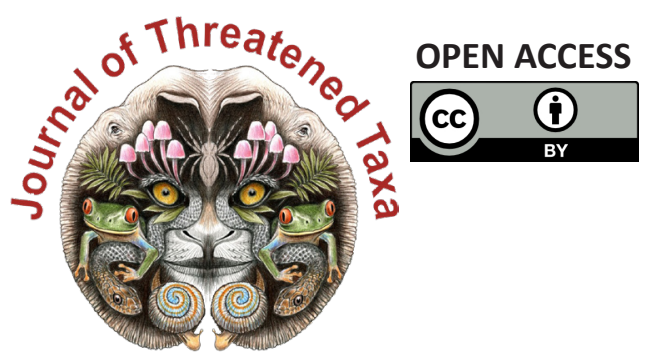

www.threatenedtaxa.org

The Journal of Threatened Taxa (JoTT) is dedicated to building evidence for conservation globally by publishing peer-reviewed articles online every month at a reasonably rapid rate at www.threatenedtaxa.org. All articles published in JoTT are registered under Creative Commons Attribution 4.0 International License unless otherwise mentioned. JoTT allows allows unrestricted use, reproduction, and distribution of articles in any medium by providing adequate credit to the author(s) and the source of publication.

ISSN 0974-7907 (Online) I ISSN $0974-7893$ (Print)

\section{November 2021 | Vol. 13 | No. 13 | Pages: 19887-20142 \\ Date of Publication: 26 November 2021 (Online \& Print) DOI: 10.11609/jott.2021.13.13.19887-20142}

\section{Article}

An inventory of geometrid moths (Lepidoptera: Geometroidea: Geometridae) of KalakadMundanthurai Tiger Reserve, India

- Geetha Iyer, Dieter Stüning \& Sanjay Sondhi, Pp. 19887-19920

\section{Communications}

Roadkills of Lowland Tapir Tapirus terrestris (Mammalia: Perissodactyla: Tapiridae) in one of its last refuges in the Atlantic Forest

- Aureo Banhos, Andressa Gatti, Marcelo Renan de Deus Santos, Leonardo Merçon,

Ilka Westermeyer, Natália Carneiro Ardente, Luis Francisco Oliveira Pereira Gonzaga, Lucas Mendes Barreto, Lucas Damásio, Tomas Lima Rocha, Vitor Roberto Schettino, Renata Valls, Helena Godoy Bergallo, Marcos Vinicius Freitas Silva, Athelson Stefanon Bittencourt, Danielle de Oliveira Moreira \& Ana Carolina Srbek-Araujo, Pp. 19921-19929

Scientific contributions and learning experiences of citizen volunteers with a small cat project in Sanjay Gandhi National Park, Mumbai, India

- Shomita Mukherjee, R. Nandini, P.V. Karunakaran \& Nayan Khanolkar, Pp. 19930-19936

Seasonal food preferences and group activity pattern of Blackbuck Antilope cervicapra (L., 1758) (Mammalia: Cetartiodactyla: Bovidae) in a semi-arid region of western Haryana, India

- Vikram Delu, Dharambir Singh, Sumit Dookia, Priya \& Kiran, Pp. 19937-19947

Studies on the habitats of Grey Francolin Francolinus pondicerianus (J.F. Gmelin, 1789) (Galliformes: Phasianidae) in northern districts of Tamil Nadu, India

- M. Pandian, Pp. 19948-19955

Recovery of vulture population in roosting and scavenging areas of Bastar and Bijapur, Chhattisgarh, India

- Sushil Kumar Dutta, Muntaz Khan, P.R.S. Nagi, Santosh Durgam \& Surabhi Dutta, Pp. 19956-19963

A geographical assessment of Chariganga and Arpara Beel (wetlands) of Nadia, West Bengal as a habitat of wetland birds

- Mehedi Hasan Mandal, Arindam Roy \& Giyasuddin Siddique, Pp. 19964-19975

Phenotypic plasticity in Barilius vagra (Hamilton, 1822) (Teleostei: Danionidae) from two geographically distinct river basins of Indian Himalaya

- Sumit Kumar, Sharali Sharma \& Deepak Singh, Pp. 19976-19984

Taxonomic notes, a new species, and a key to Indian species of the click beetle genus Cryptalaus Ôhira, 1967 (Coleoptera: Elateridae: Agrypninae)

- Harshad Parekar \& Amol Patwardhan, Pp. 19985-19999

Niche overlap of benthic macrofauna in a tropical estuary: diurnal variation

- Mário Herculano de Oliveira, Lidiane Gomes de Lima, Caroline Stefani da Silva Lima, Jéssica de Oliveira Lima Gomes, Franciely Ferreira Paiva, Graciele de Barros, Carlinda Railly Medeiros \& Joseline Molozzi, Pp. 20000-20010

Diversity of aquatic insects and biomonitoring of water quality in the upper Ganga River, a Ramsar site: a preliminary assessment

- Kritish De, Arkojyoti Sarkar, Kritika Singh, Virendra Prasad Uniyal, Jeyaraj Antony Johnson \& Syed Ainul Hussain, Pp. 20011-20018

Patterns of forest cover loss in the terrestrial Key Biodiversity Areas in the Philippines: critical habitat conservation priorities

- Bernard Peter O. Daipan, Pp. 20019-20032

The woody flora of Shettihalli Wildlife Sanctuary, central Western Ghats of Karnataka, India - A checklist

- Kanda Naveen Babu, Kurian Ayushi, Vincy K. Wilson, Narayanan Ayyappan \&

Narayanaswamy Parthasarathy, Pp. 20033-20055

Reproductive biology of Ophiorrhiza caudata C.E.C.Fisch. (Rubiaceae), an endemic and endangered creeping perennial herb of the Western Ghats, India

- Maria Theresa, Appukuttan Kamalabai Sreekala \& Jayalakshmi Mohanlal, Pp. 20056-20065
Short Communications

Successful rescue, medical management, rehabilitation, and translocation of a Red Panda Ailurus fulgens (Mammalia: Carnivora: Ailuridae) in Arunachal Pradesh, India - Jahan Ahmed, Sorang Tadap, Millo Tasser, Koj Rinya, Nekibuddin Ahmed \& Sunil Kyarong, Pp. 20066-20071

A rare photographic record of Eurasian Otter Lutra lutra with a note on its habitat from the Bhagirathi Basin, western Himalaya, India

- Ranjana Pal, Aashna Sharma, Vineet Kumar Dubey, Tapajit Bhattacharya, Jeyaraj Antony Johnson, Kuppusamy Sivakumar \& Sambandam Sathyakumar, Pp. 20072-20077

The first record of Medog Gliding Frog Rhacophorus translineatus Wu, 1977 (Anura: Rhacophoridae) from Chhukha District, Bhutan

- Sonam Lhendup \& Bal Krishna Koirala, Pp. 20078-20083

First record of a freshwater crab, Maydelliathelphusa masoniana (Henderson, 1893) (Decapoda: Brachyura: Gecarcinucidae) from West Bengal, India

- Ram Krishna Das, Pp. 20084-20089

Butterflies of Amrabad Tiger Reserve, Telangana, India

- Deepa Jaiswal, B. Bharath, M. Karuthapandi, Shrikant Jadhav, S. Prabakaran \& S. Rehanuma Sulthana, Pp. 20090-20097

An enumeration of the flowering plants of Kyongnosla Alpine Sanctuary in eastern Sikkim, India

- Sudhansu Sekhar Dash, Subhajit Lahiri \& Ashiho Asoshii Mao, Pp. 20098-20117

A new record of psychrotrophic Paecilomyces formosus (Eurotiales: Ascomycota) from India: morphological and molecular characterization

- Skarma Nonzom \& Geeta Sumbali, Pp. 20118-20123

Notes

Study on incidence and pathology of gastrointestinal parasitic infections in Nilgai Boselaphus tragocamelus in Hisar, Haryana, India

- Maneesh Sharma, B.L. Jangir, D. Lather, G.A. Chandratre, V. Nehra, K.K. Jakhar \& G. Narang, Pp. 20124-20127

An unusual vocalization of Brown Hawk-Owl Ninox scutulata (Raffles, 1822) (Aves:

Strigiformes: Strigidae) recorded from Kerala, India

- Riju P. Nair \& Shine Raj Tholkudiyil, Pp. 20128-20129

New distribution data on the genus Maripanthus Maddison, 2020 (Araneae: Salticidae) from southern India

- A. Asima, John T.D. Caleb, Dhruv A. Prajapati \& G. Prasad, Pp. 20130-20132

On the IUCN status of Boesenbergia albolutea and B. rubrolutea (Zingiberaceae) and typification of $B$. rubrolutea

- K. Aishwarya \& M. Sabu, Pp. 20133-20135

New records of mass seeding Cephalostachyum latifolium Munro (Poaceae) along the midelevation broadleaved forest of Sarpang district, Bhutan

- Jigme Tenzin, Sangay Nidup \& Dago Dorji, Pp. 20136-20139

Response

If habitat heterogeneity is effective for conservation of butterflies in urban landscapes of Delhi, India?' Unethical publication based on data manipulation

- Sanjay Keshari Das \& Rita Singh, Pp. 20140-20142

Publisher \& Host
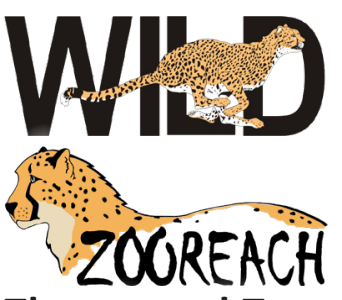

Threatened Taxa 